\title{
Is the Sokal or EUTOS long-term survival (ELTS) score a better predictor of responses and outcomes in persons with chronic myeloid leukemia receiving tyrosine-kinase inhibitors?
}

\author{
Xiao-Shuai Zhang $\mathbb{D}^{1}{ }^{1}$, Robert Peter Gale ${ }^{2}$, Xiao-Jun Huang $\mathbb{D}^{1,3,4}{ }^{凶}$ and Qian Jiang $\mathbb{D}^{1,3 凶}$ \\ (c) The Author(s) 2021
}

\begin{abstract}
Data from 1661 consecutive subjects with chronic-phase chronic myeloid leukemia (CML) receiving initial imatinib $(n=1379)$ or a $2^{\text {nd }}$ generation tyrosine-kinase inhibitor ( $2 \mathrm{G}-\mathrm{TKl} ; n=282$ ) were interrogated to determine whether the Sokal or European Treatment and Outcome Study for CML (EUTOS) long-term survival (ELTS) scores were more accurate responses and outcome predictors. Both scores predicted probabilities of achieving complete cytogenetic response (CCyR), major molecular response (MMR), failure- and progressionfree survivals (FFS, PFS), and survival in all subjects and those receiving imatinib therapy. However, the ELTS score was a better predictor of $\mathrm{MR}^{4}, \mathrm{MR}^{4.5}$, and CML-related survival than the Sokal score. In subjects receiving 2G-TKI therapy, only the ELTS score accurately predicted probabilities of CCyR, MMR, MR 4 , FFS, and PFS. In the propensity score matching, subjects classified as intermediate risk by the ELTS score receiving a 2G-TKI had better responses $(p<0.001 \sim 0.061)$, FFS $(p=0.002)$, and PFS $(p=0.03)$ but not survival. Our data suggest better overall prediction accuracy for the ELTS score compared with the Sokal score in CML patients, especially those receiving 2G-TKIs. People identified as intermediate risk by the ELTS score may benefit more from initial 2G-TKI therapy in achieving surrogate endpoints but not survival, especially when a briefer interval to stopping TKI therapy is the therapy objective.
\end{abstract}

Leukemia (2022) 36:482-491; https://doi.org/10.1038/s41375-021-01387-y

\section{INTRODUCTION}

Several risk scores have been developed to predict responses and/ or outcomes of persons with chronic-phase chronic myeloid leukemia (CML). However, predictive scores are only accurate in the context of the therapy given (as opposed to prognostic scores). For example, the Sokal and Hasford scores were developed in persons receiving chemotherapy and/or interferon $[1,2]$. The accuracy of these scores in persons receiving TKItherapy is controversial [3-6]. In contrast, the European Treatment and Outcome Study for CML (EUTOS) and EUTOS long-term survival (ELTS) scores was developed in persons receiving predominately imatinib $[7,8]$. The Sokal and ELTS scores are the most commonly used today in persons receiving TKI therapy. Several studies reported that the ELTS score is more accurate in identifying high-risk populations and better ability to predict CMLrelated deaths and survival in persons receiving imatinib or a $2^{\text {nd }}$ generation TKI (2G-TKI) [9-16]. The ELTS score is also a more accurate predictor of the probability of achieving a complete cytogenetic response (CCyR) and major molecular response (MMR) $[11,16]$. Consequently, the ELTS score is preferred in the 2020 European LeukemiaNet (ELN) recommendations [17].
Few studies critically compared the Sokal and ELTS scores as predictors of cytogenetic and molecular responses and other outcomes such as failure- and progression-free survivals (FFS and PFS), especially in persons receiving 2 G-TKIs recommended by some for persons with intermediate- or high-risk CML [18]. We compared prediction accuracies of the Sokal and ELTS scores on responses and outcomes in 1661 consecutive subjects with chronic-phase CML receiving imatinib or a 2G-TKI. We found better overall prediction accuracy for the ELTS score. People identified as intermediate risk in the ELTS score may benefit more from 2G-TKI therapy compared with imatinib in achieving surrogate endpoints but not CML-related survival or survival, especially when a briefer interval to stopping TKI therapy is the therapy objective.

\section{SUBJECTS AND METHODS}

\section{Subjects}

We interrogated data from 1661 consecutive newly diagnosed subjects with chronic-phase $C M L \geq 18$ years receiving imatinib, dasatinib, or nilotinib therapy at Peking University People's Hospital from January 2006 to March

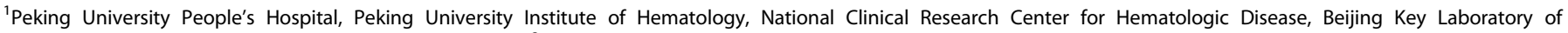

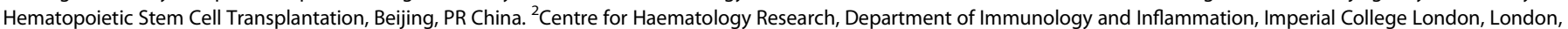

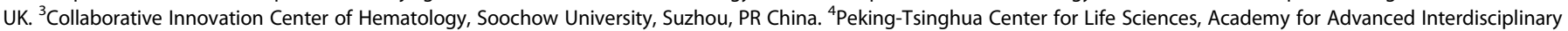
Studies, Peking University, Beijing, China. ${ }^{\bowtie}$ email: huangxiaojun@bjmu.edu.cn; jiangqian@medmail.com.cn 


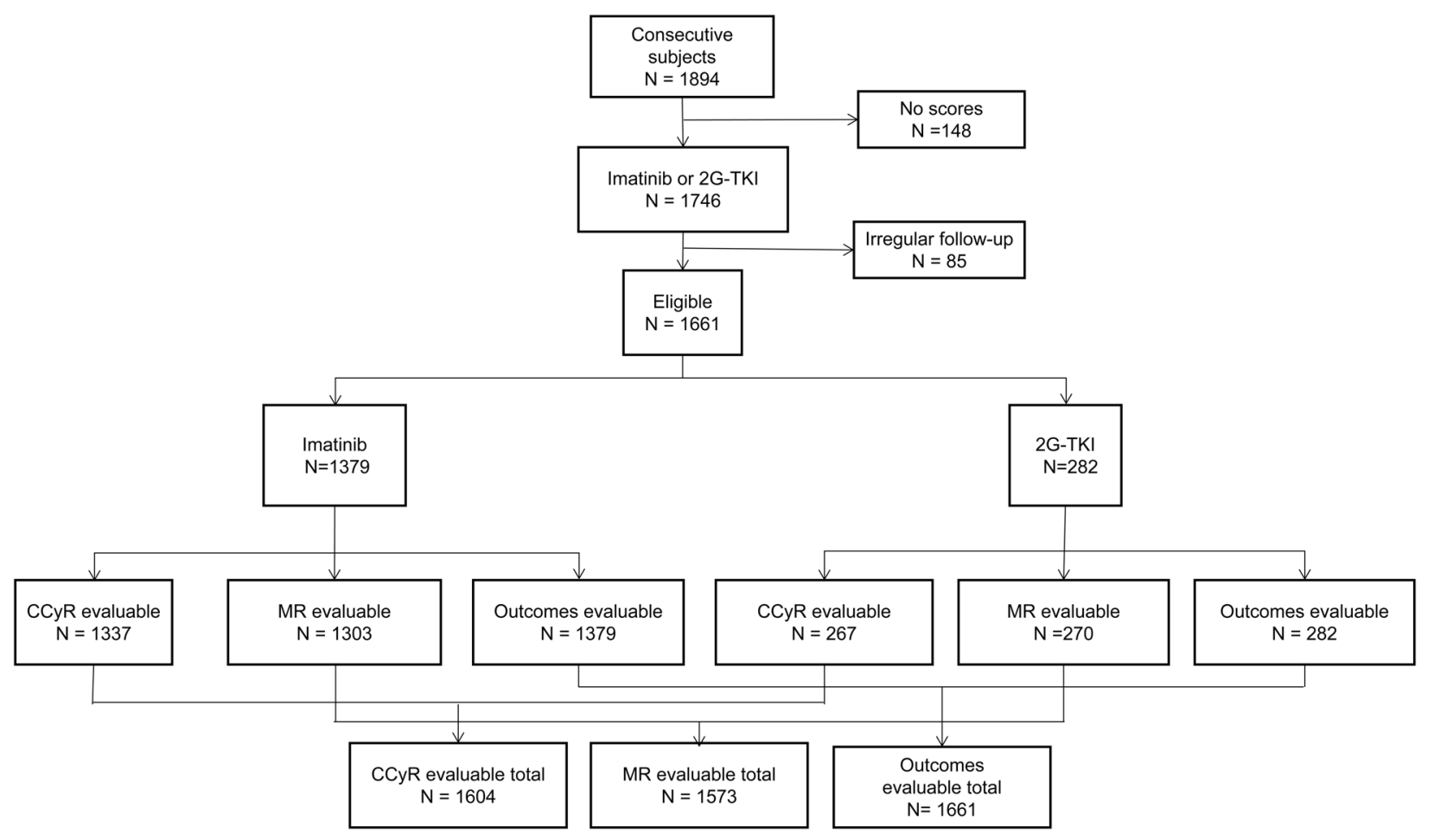

Fig. 1 Study flow diagram.

Table 1. Subject covariates.

\begin{tabular}{|c|c|c|c|c|}
\hline Variable & $\begin{array}{l}\text { All } \\
(N=1661)\end{array}$ & $\begin{array}{l}\text { Imatinib } \\
(n=1379)\end{array}$ & $\begin{array}{l}\text { 2G-TKIs } \\
(n=282)\end{array}$ & $p$-value \\
\hline Sex & & & & 0.721 \\
\hline Sokal risk & & & & $<0.001$ \\
\hline Low & 755 (45.5\%) & $651(47.2 \%)$ & $104(36.9 \%)$ & \\
\hline ELTS risk & & & & $<0.001$ \\
\hline Low & 1098 (66.1\%) & 937 (67.9\%) & $161(57.1 \%)$ & \\
\hline Intermediate & $414(24.9 \%)$ & $334(24.2 \%)$ & $80(28.4 \%)$ & \\
\hline High & 149 (9.0\%) & $108(7.8 \%)$ & 41 (14.5\%) & \\
\hline WBC, $\times 10 E+9 / L$ & $120(3,786)$ & $112(5,786)$ & $155(5,755)$ & 0.001 \\
\hline Blood basophils, \% & $4(0,19)$ & $4(0,19)$ & $5(0,19)$ & 0.003 \\
\hline $\mathrm{Ph}^{+} \mathrm{ACAs}$ & & & & 0.257 \\
\hline Yes & $50(3.0 \%)$ & $36(2.6 \%)$ & $14(5.0 \%)$ & \\
\hline No & 1042 (84.4\%) & 1163 (84.3\%) & $239(84.8 \%)$ & \\
\hline Unknown & $209(12.6 \%)$ & $180(13.1 \%)$ & $29(10.3 \%)$ & \\
\hline$\geq 1$ Co-morbidity(ies) & 605 (36.4\%) & $510(37.0 \%)$ & 95 (33.7\%) & 0.295 \\
\hline Follow-up, months & $58(3,193)$ & $60(3,193)$ & $46(3,160)$ & $<0.001$ \\
\hline
\end{tabular}

The data are presented as the number (\%) or median (range), except where otherwise noted.

2G-TKI second-generation tyrosine kinase inhibitor, $\mathrm{Ph}^{+}$ACA additional chromosomal aberrations in Philadelphia-positive cells, PLT platelet, TKI tyrosine kinase inhibitor, WBC white blood cell. 

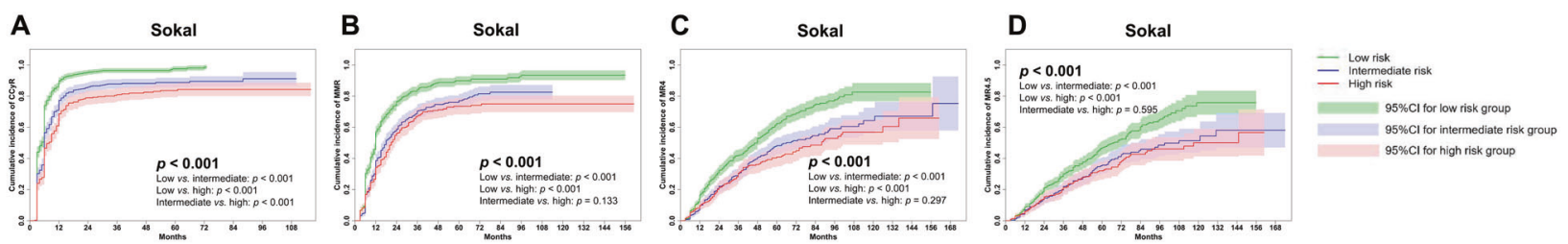

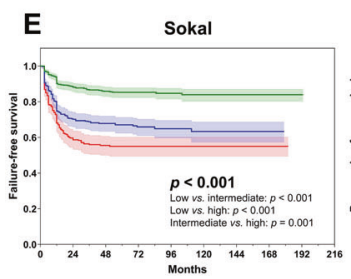

F Sokal

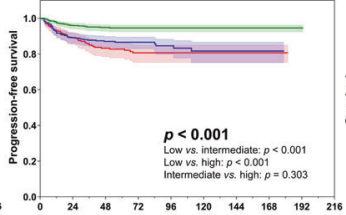

G

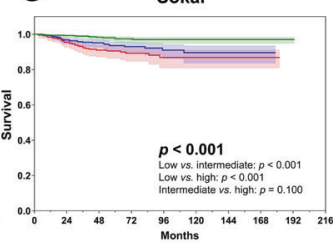

K
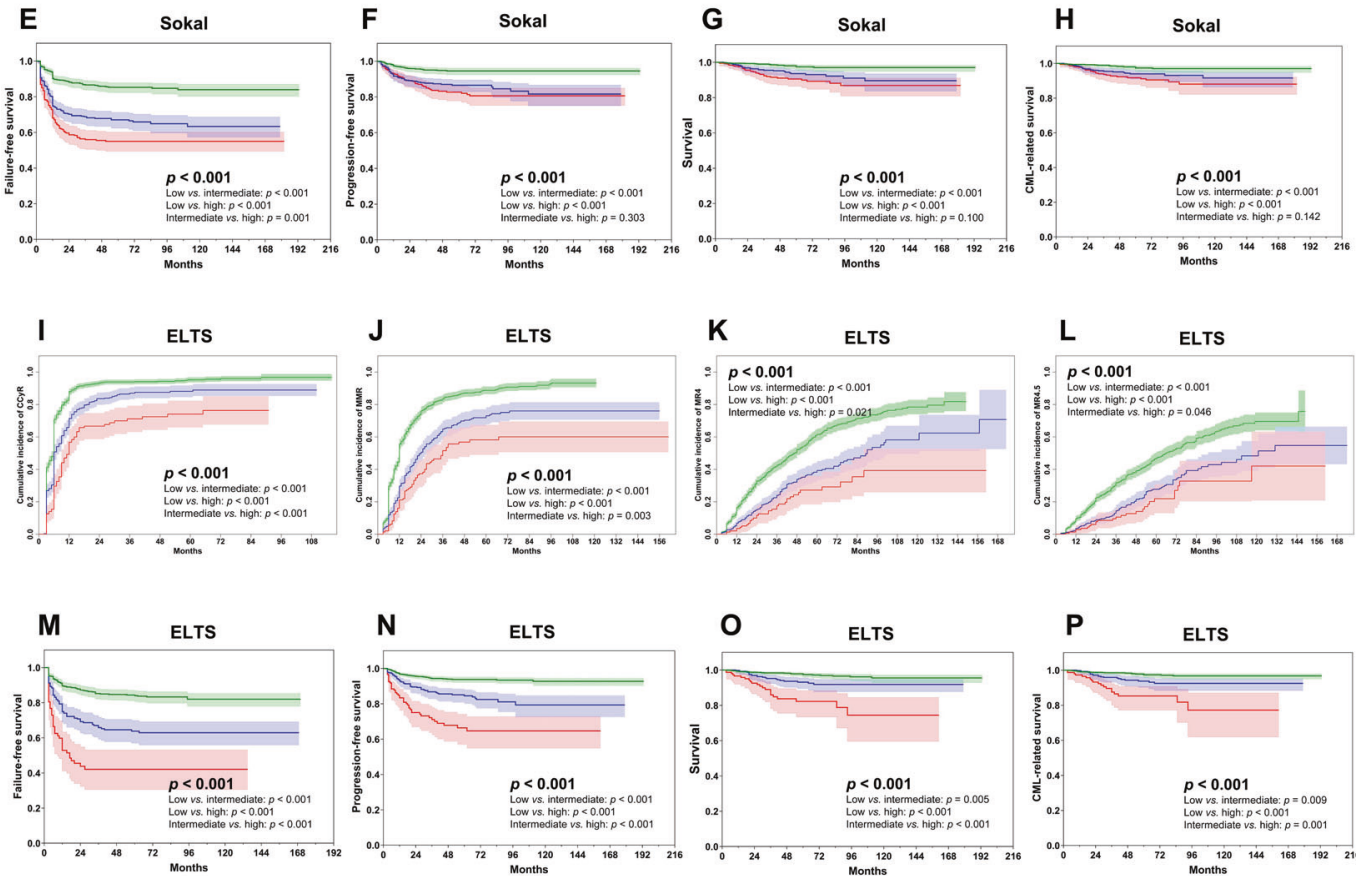

Fig. 2 Therapy responses and outcomes of all subjects. A-D CCyR, MMR, MR ${ }^{4}$, and $M R^{4.5}$ by Sokal score, E-H FFS, PFS, survival, and CMLrelated survival by Sokal score, I-L CCyR, MMR, MR ${ }^{4}$, and $\mathrm{MR}^{4.5}$ by ELTS score, M-P FFS, PFS, survival, and CML-related survival by ELTS score.

2021. Data of covariates determined at diagnosis included sex, age, comorbidities, hemoglobin concentration, WBC and platelet counts, cytogenetic analyses, and initial TKI therapy. Sokal and ELTS scores at diagnosis were calculated as described $[2,8]$. Therapy responses and outcomes were extracted from medical records. Physicians and patients jointly choose the initial TKI given based on which TKIs were available, anticipated safety and efficacy, and economics. The initial imatinib dose was $400 \mathrm{mg}$ daily; nilotinib, $300 \mathrm{mg}$ twice daily; dasatinib, $100 \mathrm{mg}$ daily. Dose and/or type of TKI were adjusted during therapy based on responses, adverse events, and operative ELN recommendations [17, 19-21]. The study was approved by the Ethics Committee of Peking University People's Hospital compliant with the Helsinki Declaration. Subjects gave written informed consent.

\section{Diagnosis, monitoring, responses, and outcomes}

Diagnosis, monitoring, and therapy responses conformed operative ELN recommendations [17, 19-21]. Bone marrow cytogenetic analyses used G-banding. $B C R: A B L 1$ transcript levels in blood were assessed by quantitative real time polymerase chain (qRT-PCR) with $A B L 1$ as control and converted to international scales $\left(B C R:: A B L 1^{15}\right)$ using our laboratoryspecific conversion factor of 0.65 (Institute of Medical and Veterinary Science International Reference Laboratory, Adelaide, Australia) [22].

Response assessment was performed on the intention-to-treat population. Haematologic response was monitored every 1-2 weeks, until a complete hematologic response (CHR) and every 3-6 months thereafter. The cytogenetic response was assessed at baseline and then every 3-6 months, until a CCyR was achieved and repeated at therapy failure. High-risk additional cytogenetic abnormalities (ACAs) were defined according to 2020 ELN criteria [17]. Molecular monitoring was done at baseline and every 3 months, until major molecular response (MMR) and every 3-6 months thereafter. Screening for $A B L 1$ mutation was done in subjects with a suboptimal or warning response according to operative ELN criteria [17, 19-21].

Responses and outcomes were defined as follows: (1) CCyR, no Ph [1]positive cells in $\geq 20$ bone marrow cell metaphases; (2) MMR, BCR::ABL1 $1^{15} \leq$
0.1\%; (3) molecular response $4\left(M^{4}\right), B C R:: A B L 1^{15} \leq 0.01 \%$; (4) molecular response $4.5\left(\mathrm{MR}^{4.5}\right), B C R:: A B L 1^{15} \leq 0.0032 \%$; (5) therapy failure, according to the 2020 ELN criteria, including failing to meet the ELN time-dependent $B C R:: A B L 1$ transcript levels, development of $A B L 1$ mutations and/or highrisk additional cytogenetic abnormalities, or progression to accelerated and/or blast phases [17].

\section{Statistical analyses}

Descriptive statistics were used to summarize covariates. Categorical variables are reported as percentages and counts and continuous variables as medians and ranges. Pearson chi-squared test (for categorical variables) and Mann-Whitney $\mathrm{U}$ test (for continuous variables) were used to compare the imatinib and 2G-TKI cohorts. Cumulative incidences of CCYR, MMR, $\mathrm{MR}^{4}$, and $\mathrm{MR}^{4.5}$ were calculated using the Fine-Gray test that considered competing events such as death, transplant, loss to follow-up, and/or withdrawal of consent. Failure- and progression-free survivals (FFS, PFS), CML-related survival, and survival were calculated using the Kaplan-Meier estimator and log-rank tests.

Potential predictive covariates for diverse responses and outcomes were tested in univariable analyses and those with $p<0.2$ were included in multivariable analyses using a backward-elimination process to fit a Cox regression model. Cox regression models were built to identify independent covariates associated with responses and outcomes reported as hazard ratios (HRs) with $95 \%$ confidence intervals (Cls).

FFS was calculated from TKI-therapy start to therapy failure or censored at the last follow-up. PFS was calculated as TKI-therapy start to progression, death at any time, or censored at the last follow-up. CML-related survival was calculated from TKI-therapy start to death from CML progression or censored at the last follow-up. Survival was calculated as TKI therapy to death from any cause or censored at the last follow-up.

Propensity-score matching was used to explore whether the Sokal or ELTS score was a better predictor of responses and outcomes to imatinib or $2 \mathrm{G}-\mathrm{TKI}$ as $1^{\text {st }}$ therapy, including all covariates tested in the univariable 

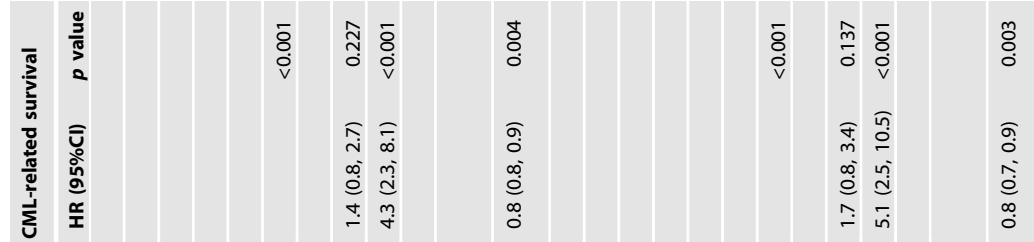

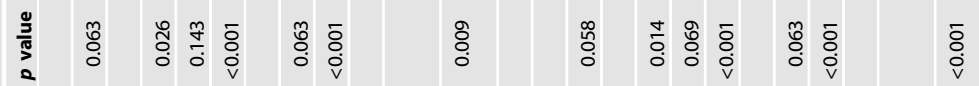

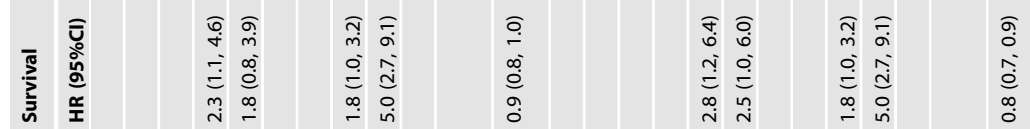

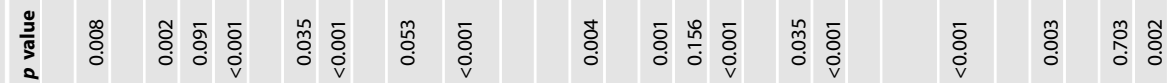

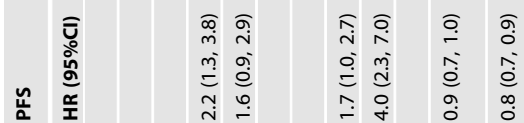

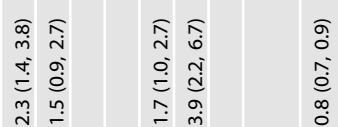

ฐิ

นิ่

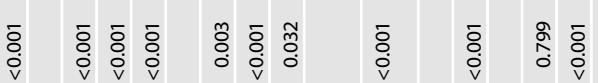

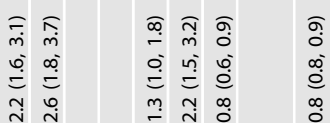

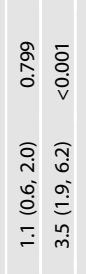

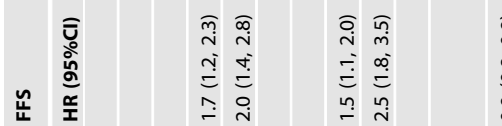

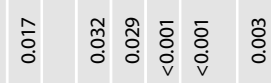

ㅎำ ฮำ

흠

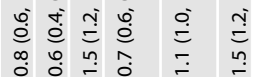

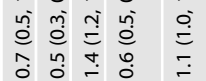

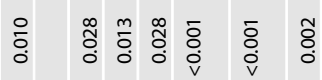

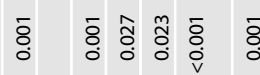

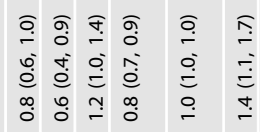

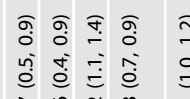

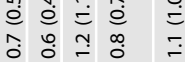

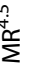

站

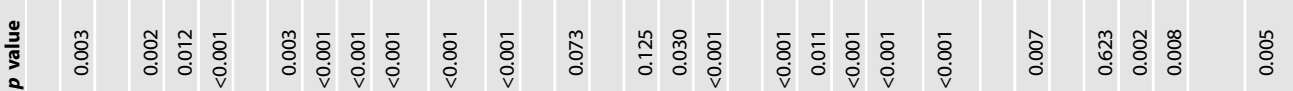

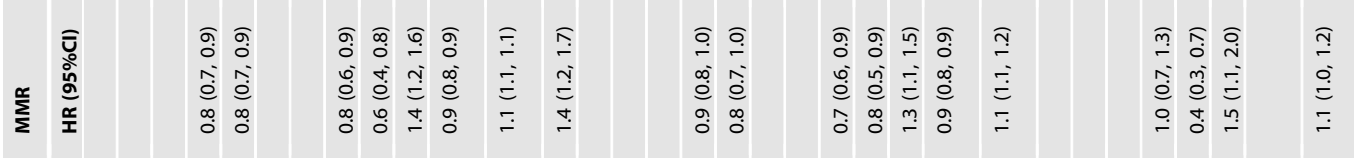

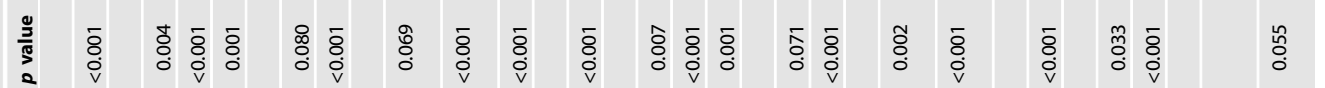

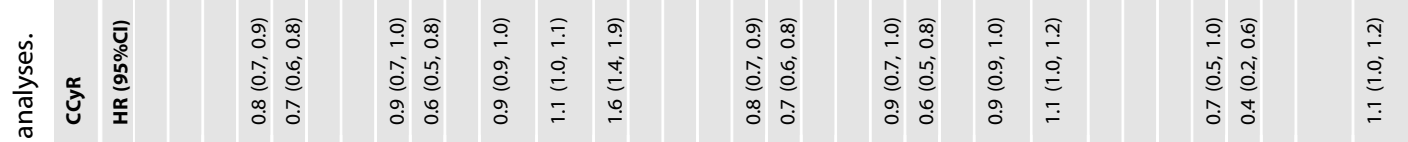

$\frac{\frac{0}{0}}{\frac{0}{0}}$

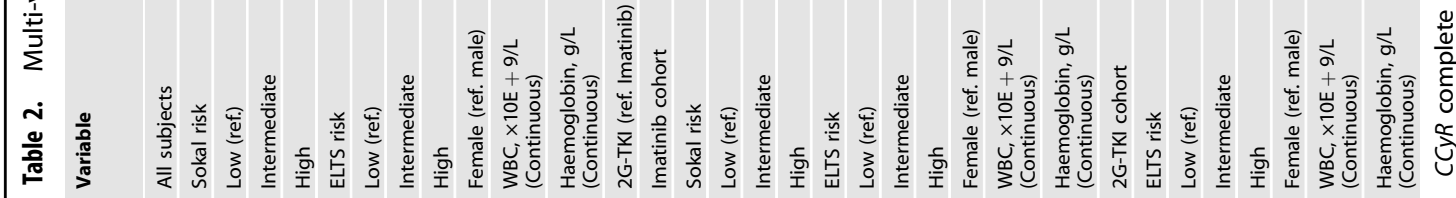



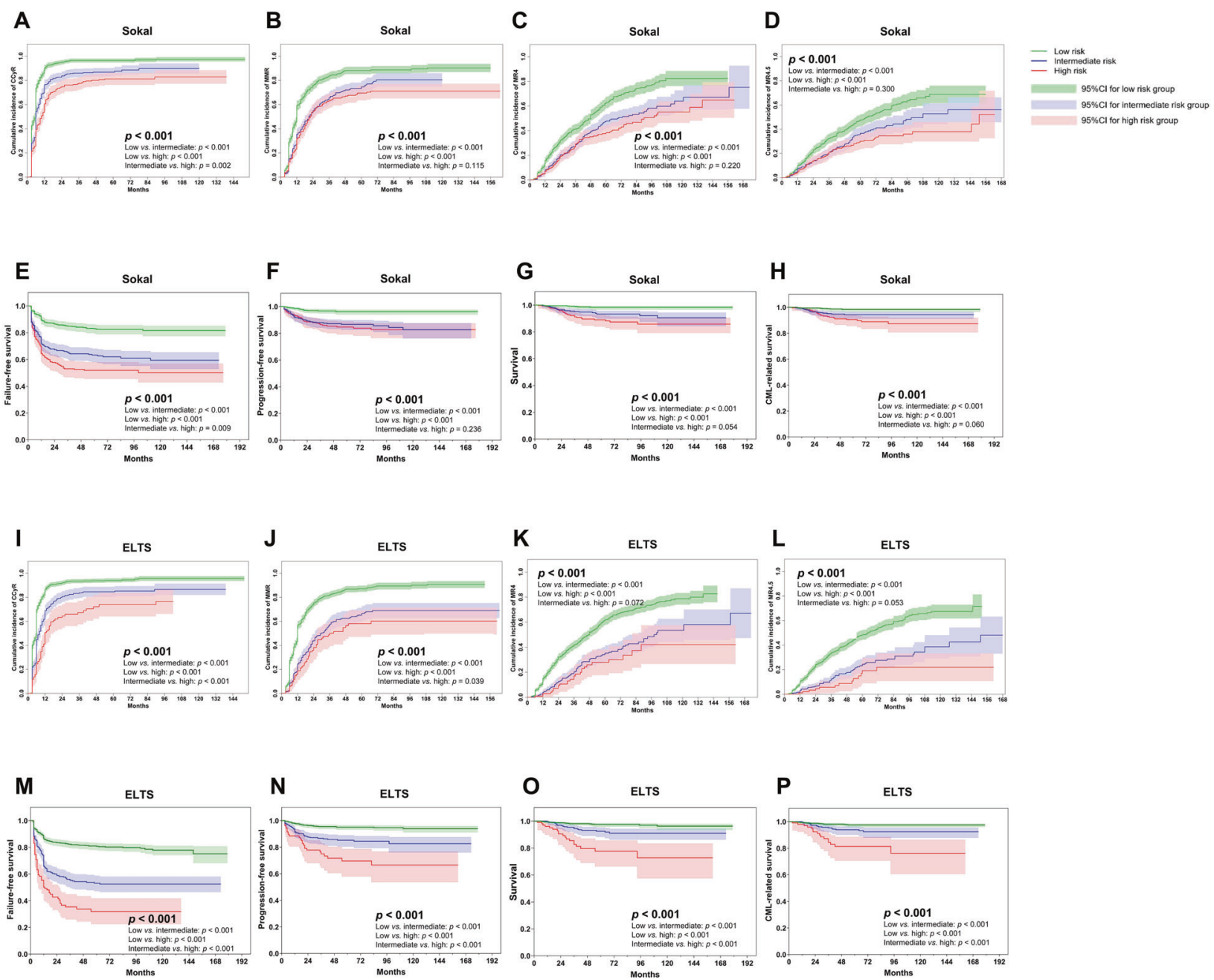

Fig. 3 Therapy responses and outcomes of subjects receiving initial imatinib. A-D CCyR, MMR, MR ${ }^{4}$, and MR ${ }^{4.5}$ by Sokal score, E-H FFS, PFS, survival, and CML-related survival by Sokal score, I-L CCyR, MMR, MR ${ }^{4}$, and MR $^{4.5}$ by ELTS score, M-P FFS, PFS, survival, and CML-related survival by ELTS score.

analyses. Covariate balance was evaluated using the standardized absolute mean difference (SAMD). SAMD $<0.02$ was considered adequate balance. A two-sided test with $p<0.05$ was considered statistically significant. SPSS 22.0 (SPSS, Chicago, IL, USA), R version 4.0.2 (R Core Team, Vienna, Austria), and GraphPad Prism 8 (GraphPad Software Inc., La Jolla, CA, USA) were used for analyses and graphing.

\section{RESULTS}

\section{Subjects}

In total, 1894 consecutive subjects were interrogated. In total, 233 were excluded because there were no Sokal and ELTS scores $(n=$ $148)$ and irregular follow-up $(n=85)$. The 1661 remaining subjects initially received imatinib $(n=1379 ; 83 \%)$, nilotinib $(n=206 ; 12 \%)$, or dasatinib ( $n=76 ; 5 \%$; Fig. 1$) ; 1021$ subjects $(62 \%)$ were male. Median age was 40 years (interquartile range [IQR], 29-51 years). In total, 605 (36\%) had $\geq 1$ comorbidity and 18, $\geq 1$ high-risk ACAs. In total, 755 (46\%), 522 (31\%), and $384(23 \%)$ were classified as low-, intermediate-, or high risk using the Sokal score. Similar assignments using the ELTS score were 1098 (66\%), 414 (25\%), and $149(9 \%)$. In total, 1633 subjects (98\%) had e13a2 and/or e14a2 BCR::ABL1 and 28 subjects (2\%), other transcripts.

Subjects initially receiving a 2G-TKI therapy were younger $(p<0.001)$, had the higher WBC counts $(p=0.001)$, lower hemoglobin concentration $(p<0.001)$, higher percentages of blood blasts $(p=0.001)$, and basophils $(p=0.003)$, and were more likely high-risk using Sokal and ELTS scores ( $p<0.001$; Table 1). Median follow-up is 60 months (IQR, 33-85 months) in the imatinib cohort and 46 months (IQR, 19-68 months) in the 2G-TKI cohort $(p<0.001)$ because of the later availability of $2 \mathrm{G}-\mathrm{TKI}$. In total, 1074 subjects (78\%) in the imatinib cohort and 247 in the $2 \mathrm{G}-\mathrm{TKI}$ cohort remained on their 1 st TKI ( $79 \%$ vs. $88 \% ; p=0.18)$. In total, 298 subjects $(22 \%)$ receiving initial imatinib switched to nilotinib $(n=224)$, dasatinib $(n=66)$, or, olverembatinib $(n=8)$ as their 2 nd $(n=257)$ or 3 rd $(n=$ 33), TKI because of therapy failure $(n=242)$, adverse events $(n=33)$ or by choice $(n=23)$. 33 subjects $(12 \%)$ receiving initial 2G-TKI therapy switched to imatinib $(n=27)$ or olverembatinib $(n=6)$ as 2nd $(n=31)$ or 3rd $(n=2)$ TKI because of cost $(n=17)$, adverse events $(n=12)$, or therapy failure $(n=4)$. In total, 9 subjects receiving initial imatinib $(n=7)$ or $2 \mathrm{G}-\mathrm{TKI}(n=2)$ discontinued TKI therapy after achieving $\geq \mathrm{MR}^{4}$. In total, 19 subjects were lost to follow-up totally.

\section{All subjects}

In total, 1614 subjects (97\%) achieved a CHR. In total, 1604 subjects (97\%) were studied for CCyR. In total, 1573 with common $B C R$ :: $A B L 1$ transcripts were studied for MMR, $\mathrm{MR}^{4}$, and $\mathrm{MR}^{4.5}$. In total, 1464 (91\% [95\% Confidence Interval (Cl), 87, 95\%]), 1208 (77\% [73, $81 \%]), 1070(68 \%$ [63, 74\%]), and 1002 (64\% [58, 70\%]) achieved a CCyR, MMR, MR ${ }^{4}$, and $\mathrm{MR}^{4.5}$. In total, 419 subjects $(25 \%[22,28 \%])$ had therapy failure, $156(9 \%[7,11 \%])$ transformed to accelerated 

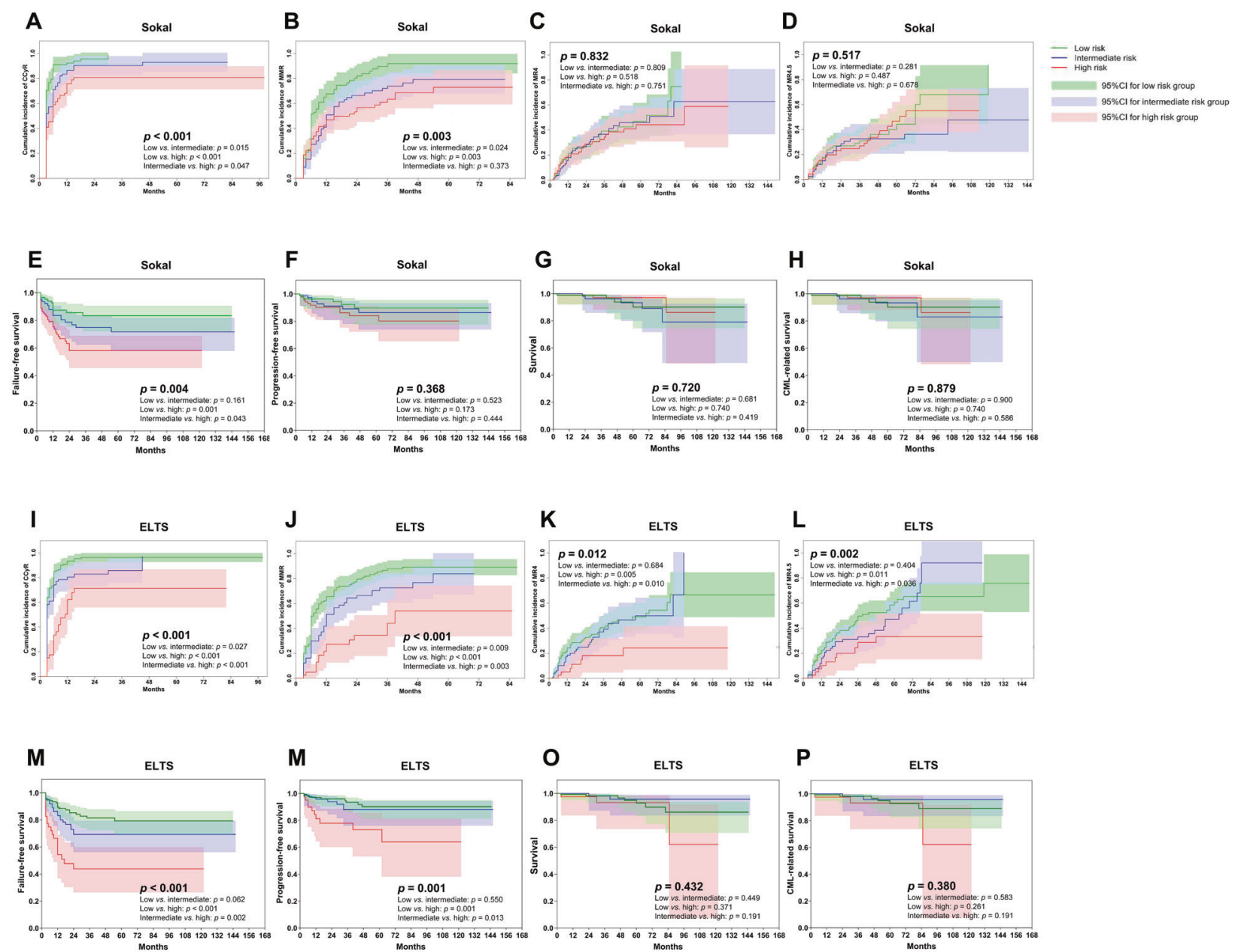

Fig. 4 Therapy responses and outcomes of subjects receiving initial 2G-TKIs. A-D CCyR, MMR, MR ${ }^{4}$, and MR ${ }^{4.5}$ by Sokal score, E-H FFS, PFS, survival and CML-related survival by Sokal score, I-L CCyR, MMR, MR ${ }^{4}$, and MR $^{4.5}$ by ELTS score, M-P FFS, PFS, survival, and CML-related survival by ELTS score.

$(n=88,5 \%[4,6 \%])$ or blast phases $(n=68,4 \%[3,5 \%])$, and 75 subjects $(5 \%[3,6 \%])$ died of transformation to accelerated or blast phases $(n=68,4 \%[3,5 \%])$ or other causes $(n=7,<1 \%)$. In all, 7-year cumulative incidences of CCyR, MMR, MR ${ }^{4}$, and $\mathrm{MR}^{4.5}$ were $92 \%(87,96 \%), 83 \%(77,91 \%), 65 \%(61,69 \%)$, and 54\% (39, $68 \%)$. About 7-year probabilities of FFS, PFS, CML-related survival, and survival were $72 \%(70,74 \%), 89 \%(87,92 \%), 94 \%(92,95 \%)$, and 94\% (92, 95\%; Fig. 2).

Subject covariates indicated above were analyzed to explore whether the Sokal or ELTS score was a better predictor of CCyR, $M M R, M^{4}, M R^{4.5}, F F S, P F S$, survival, and CML-related survival. There were no interactions between these covariates (VIFs = 1.0-1.7). In multivariable analyses, both scores were significantly associated with the probabilities of CCyR (Sokal, $p<0.001$; ELTS, $p=0.001$ ), MMR (Sokal, $p=0.003$; ELTS, $p<0.001$ ), FFS (Sokal, $p<$ 0.001; ELTS: $p<0.001$ ), PFS (Sokal, $p=0.008$; ELTS, $p<0.001$ ), and survival (Sokal, $p=0.063$; ELTS, $p<0.001$ ). However, only the ELTS score was significantly associated with the cumulative incidence of $\mathrm{MR}^{4}$ (intermediate vs. low, hazard ratio $[\mathrm{HR}]=0.8$ $[0.6,1.0], p=0.028$; high vs. low, $\mathrm{HR}=0.6[0.4,0.9], p=0.013$ ), $\mathrm{MR}^{4.5}$ (intermediate vs. low, $\mathrm{HR}=0.8[0.6,1.0], p=0.041$; high vs. low, $\mathrm{HR}=0.6[0.4,0.9], p=0.030$ ), and CML-related survival (high vs. low, $\mathrm{HR}=4.3[2.3,8.1], p<0.001)$. Male sex, lower hemoglobin concentration, higher WBC counts, and initial imatinib therapy were significantly associated with lower probabilities of molecular responses and/or inferior outcomes (Table 2).

\section{Imatinib cohort}

In total, 1337 of 1379 subjects (97\%) receiving initial imatinib were studied CCyR.1303 with common $B C R:: A B L 1$ transcripts were studied for MMR, MR ${ }^{4}$, and $\mathrm{MR}^{4.5} .1221$ (91\% [88, 94\%]), 1003 $(77 \%[73,80 \%]), 928(71 \%[66,74 \%])$, and $889(68 \%[63,72 \%])$ achieved a CCyR, MMR, MR ${ }^{4}$, and $\mathrm{MR}^{4.5}$. In total $349(26 \%[23$, $28 \%])$ had therapy failure, $127(9 \%[8,11 \%])$ transformed to accelerated $(n=67,5 \%[4,6 \%])$ or blast phases $(n=60,4 \%[3$, $5 \%])$, and 62 subjects $(5 \%[3,6 \%])$ died of $C M L$ transformation to accelerated or blast phases $(n=56,4 \%[3,5 \%])$ or other causes ( $n=6,<1 \%$ ). About 7-year cumulative incidences of CCyR, MMR, $\mathrm{MR}^{4}$, and $\mathrm{MR}^{4.5}$ were $90 \%(85,96 \%), 78 \%(74,83 \%), 54 \%(45,66 \%)$, and $43 \%(39,54 \%)$. About 7 -year probabilities of FFS, PFS, CMLrelated survival and survival were $70 \%(65,77 \%), 89 \%(83,94 \%)$, 95\% (92, 97\%), and 94\% (91, 97\%; Fig. 3).

In multivariable analyses, both scores were significantly associated with the probabilities of CCyR (Sokal, $p<0.001$; ELTS, $p=0.001$ ), MMR (Sokal, $p=0.073$; ELTS, $p<0.001$ ), FFS (Sokal, $p<0.001$; ELTS: $p<0.001$ ), PFS (Sokal, $p=0.004$; ELTS, $p<0.001$ ), and survival (Sokal, $p=0.058$; ELTS, $p<0.001$ ). However, only the ELTS score was significantly associated with the cumulative incidence of $\mathrm{MR}^{4}$ (intermediate vs. low, $\mathrm{HR}=0.7[0.5,0.9]$, $p=0.001$; high vs. low, $\mathrm{HR}=0.6[0.4,0.9], p=0.027), \mathrm{MR}^{4.5}$ (intermediate vs. low, $\mathrm{HR}=0.7[0.5,1.0], p=0.032$; high vs. low, $\mathrm{HR}=0.5[0.3,0.9], p=0.029$ ), and CML-related survival (high vs. low, $\mathrm{HR}=5.1[2.5,10.5], p<0.001)$. Male sex, lower hemoglobin 
Table 3. Covariates in propensity score-matched cohorts.

\begin{tabular}{|c|c|c|c|c|}
\hline Variable & All $(N=1332)$ & Imatinib $(n=1064)$ & 2G-TKIs $(n=268)$ & $p$-value \\
\hline Age, years & $38(18,80)$ & $38(18,80)$ & $38(18,73)$ & 0.664 \\
\hline Sex & & & & 0.878 \\
\hline Male & 791 (59.4\%) & $625(58.7 \%)$ & $165(61.7 \%)$ & \\
\hline Sokal risk & & & & 0.307 \\
\hline Low & $524(39.2 \%)$ & $429(40.3 \%)$ & 95 (35.4\%) & \\
\hline Intermediate & $446(33.5 \%)$ & $361(33.9 \%)$ & $85(31.7 \%)$ & \\
\hline ELTS risk & & & & 0.153 \\
\hline Low & $806(60.5 \%)$ & $654(61.5 \%)$ & $153(57.2 \%)$ & \\
\hline Intermediate & $389(29.2 \%)$ & $311(29.2 \%)$ & 78 (29.1\%) & \\
\hline High & $137(10.3 \%)$ & 99 (9.3\%) & 37 (13.7\%) & \\
\hline WBC, $\times 10 E+9 / L$ & $148(3,786)$ & $145(5,786)$ & $163(6,755)$ & 0.287 \\
\hline $\mathrm{Ph}^{+} \mathrm{ACA}$ & & & & 0.892 \\
\hline Yes & $40(3.0 \%)$ & $32(3.0 \%)$ & $8(3.0 \%)$ & \\
\hline$\geq 1$ Comorbidity(ies) & $463(34.8 \%)$ & 375 (35.2\%) & $88(32.8 \%)$ & 0.620 \\
\hline Follow-up months & $54(3,178)$ & $55(3,178)$ & $45(3,164)$ & $<0.001$ \\
\hline
\end{tabular}

The data are presented as the number (\%) or median (range), except where otherwise noted.

2G-TKI second-generation tyrosine kinase inhibitor, $\mathrm{Ph}^{+}$ACA additional chromosomal aberrations in Philadelphia-positive cells, PLT platelet, TKI tyrosine kinase inhibitor, WBC white blood cell.

concentration, and higher WBC counts were significantly associated with lower probabilities of molecular responses and/or inferior outcomes (Table 2).

\section{G-TKI cohort}

In total, 267 of 282 subjects (95\%) receiving initial 2G-TKI were studied for CCyR. In total, 270 with common $B C R:: A B L 1$ transcripts were studied for MMR, MR ${ }^{4}$, and $\mathrm{MR}^{4.5}$. In total, 243 (91\% [85, 97\%]), 205 (76\% [69, 82\%]), 142 (53\% [46, 60\%]), and $113(40 \%[34,46 \%])$ achieved CCyR, MMR, MR ${ }^{4}$, and $M^{4.5}$. In total, $70(25 \%[20,30 \%])$ had therapy failure, $29(10 \%[7,14 \%])$ transformed to accelerated $(n=21,7 \%[6,8 \%])$ or blast phases $(n=8 ; 3 \%[2,4 \%])$, and $13(5 \%[2,7 \%])$ died of CML progression $(n=12,4 \%[2,7 \%])$ or other causes $(n=1,<1 \%)$. About 5 -year cumulative incidences of CCyR, MMR, MR ${ }^{4}$, and $\mathrm{MR}^{4.5}$ were $91 \%$ $(86,97 \%), 78 \%(67,84 \%), 58 \%(49,67 \%)$, and $42 \%(30,59 \%)$. About 5-year probabilities of FFS, PFS, CML-related survival, and survival were $71 \%(57,84 \%), 87 \%(72,96 \%), 92 \%(71,98 \%)$, and 91\% (71, 99\%; Fig. 4).

In multivariable analyses, only the ELTS score predicted probabilities of CCyR (intermediate vs. low, $\mathrm{HR}=0.7[0.5,1.0]$; $p=0.033$; high vs. low, $\mathrm{HR}=0.4[0.2,0.6], p<0.001$ ), MMR (high vs. low, $\mathrm{HR}=0.4[0.3,0.7], p=0.002$ ), and $\mathrm{MR}^{4}$ (high vs. low, $\mathrm{HR}=$ $0.4[0.2,0.9], p=0.024$ ), as well as worse FFS (high vs. low, $\mathrm{HR}=$ $3.5[1.9,6.2], p<0.001$ ), and PFS (high vs. low, HR=4.1 [1.7, 9.9]; $p=0.002)$. However, the Sokal score did not accurately predict responses or outcomes. Male sex, lower hemoglobin concentration, and higher WBC counts were significantly associated with lower probabilities of molecular responses and/or worse outcomes (Table 2).

\section{Is the sokal or ELTS score a better predictor of response and} outcomes?

Because of significant differences in baseline covariates between the imatinib and 2G-TKI cohorts, we used propensity-score matching to adjust subjects. In total 1332 matches were identified in the imatinib $(n=1064 ; 80 \%)$ and 2G-TKI $(n=268 ; 20 \%)$ cohorts (Table 3).

Median follow-up was 55 months (IQR, 30-85 months) in the imatinib cohort and 46 months (IQR, 20-64 months; $p<0.001)$ in the 2G-TKI cohort. There were no significant differences in FFS, PFS, CML-related survival, or survival in the low- and high-risk cohorts defined by either the Sokal or ELTS scores whether subjects received initial imatinib or a 2G-TKI, except for probabilities of cytogenetic and/or molecular responses (Supplementary Figs. 1-2). However, in the intermediate-risk cohort defined by either the Sokal or ELTS scores, subjects receiving initial 2G-TKI therapy had higher probabilities of CCyR, MMR, and MR ${ }^{4.5}$ and a better FFS compared with those receiving initial imatinib. Better MR ${ }^{4}$ and PFS were detected only with the ELTS score $(p<$ 0.001 and $p=0.032$ ). However, initial TKI therapy had no impact on CML-related survival or survival using either the Sokal or ELTS scores (Fig. 5). We did not analyze interval to stopping TKI therapy or success rate of therapy-free remission.

\section{DISCUSSION}

We compared predictive accuracies of the Sokal and ELTS scores in 1661 subjects with chronic-phase CML. We found that the ELTS score was a better overall response and outcome predictor, especially in subjects receiving initial 2G-TKI therapy. Based on $\mathrm{HRs}$ and $\mathrm{Cls}$ in multivariable analyses, the ELTS score was a better discriminator between risk cohorts than the Sokal score.

Our data are consistent with some previous findings [10-16]. The study by Pfirrmann and colleagues reported that the ELTS was a better survival predictor than the Sokal score $[8,14]$. However, our study focused on FFS rather than survival. As such, it is more likely to be of use to physicians in choosing the best initial TKI therapy. Geelen et al. reported that the ELTS score identified significant differences in probabilities of MMR, CML-related death, 

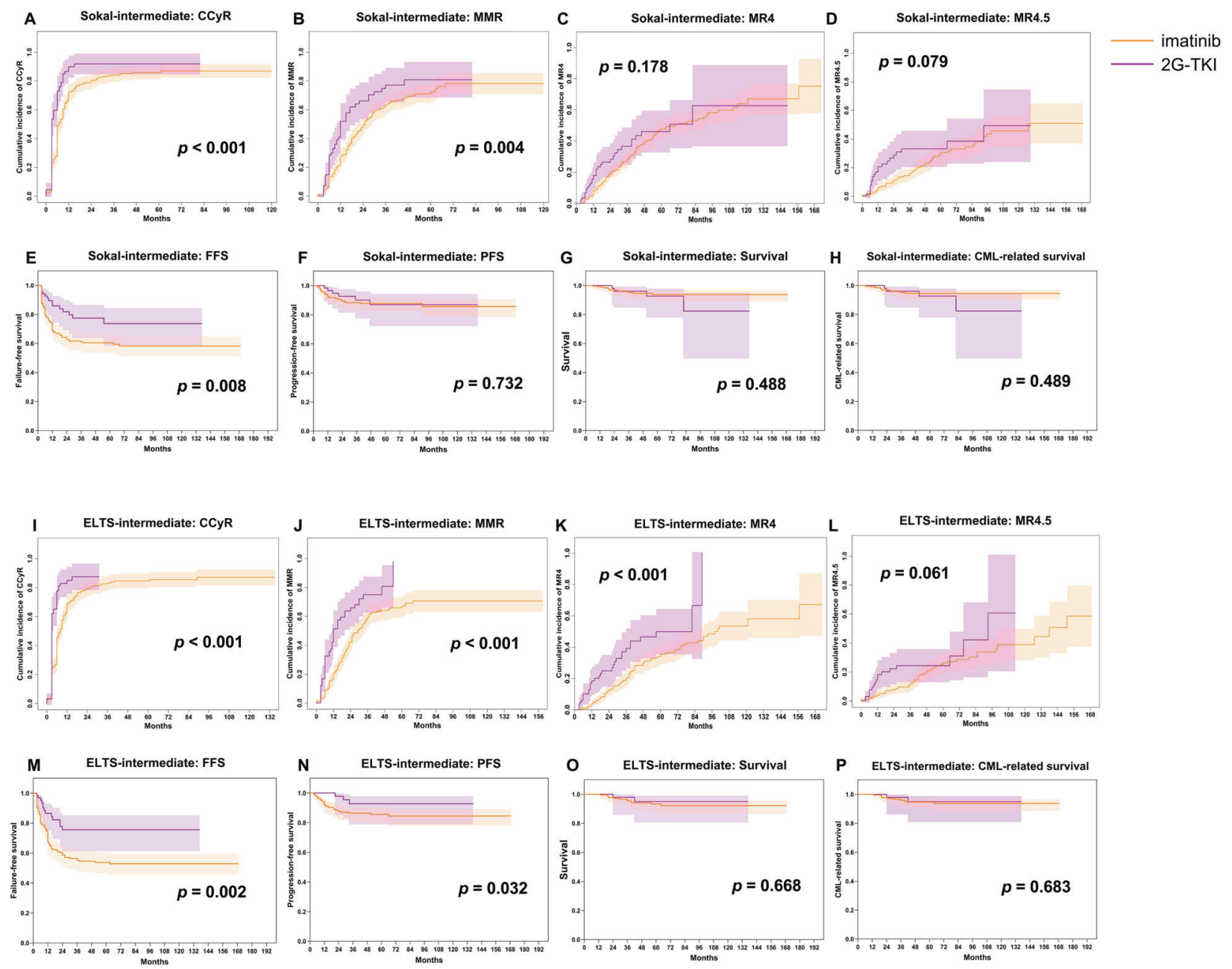

Fig. 5 Therapy responses and outcomes in intermediate-risk subjects after the propensity-score matching. A-D CCyR, MMR, MR ${ }^{4}$, and $\mathrm{MR}^{4.5}$ by Sokal score, E-H FFS, PFS, CML-related survival, and survival by Sokal score, I-L CCyR, MMR, MR ${ }^{4}$, and MR ${ }^{4.5}$ by ELTS score, M-P FFS, PFS, survival, and CML-related survival by ELTS score.

and survival in subjects receiving 2G-TKls compared with the Sokal score [11]. We found that the ELTS score predicted probabilities of CCyR, MMR, MR ${ }^{4}$, FFS, and PFS in subjects receiving initial $2 \mathrm{G}-\mathrm{TKI}$ therapy but not $\mathrm{MR}^{4.5}$. However, the ELTS score was not predictive of CML-related survival or survival. Discordances between our data and those of Geelen et al. might result from the younger age of our subjects, which is an independent predictive covariate for survival in many studies [9-16]. Also, these studies may not have been comparable for therapies given after initial 2G-TKI therapy. It is not surprising that the ELTS score is a better predictor of responses and outcomes of TKI therapy, because it was derived from a dataset of subjects receiving TKI therapy whereas the Sokal score was developed in a dataset of subjects receiving other therapies. As such, the Sokal score is best considered prognostic rather than predictive score better reflecting $\mathrm{CML}$ biology than therapy.

We found fewer non-CML-related deaths compared with other studies [9-16]. There are several possible explanations, including the younger age of our subjects who would be expected to be otherwise healthier, have fewer comorbidities, and therefore fewer competing causes of death [23, 24]. Also, as a tertiary referral center, there are likely subject-selection biases. For example, persons with substantial other health problems were less likely to travel to our center.

One potentially problematic area is defining failure. In our literature review, we found no consistent definition. We used definitions proposed in the 2020 ELN CML recommendations [17]. Because there was no consensus definition of accelerated phase, we analyzed our data including and excluding subjects in whom progression to accelerated phase was the failure event. Our conclusions were unchanged.

Several studies report that initial 2G-TKIs are associated with faster cytogenetic and molecular responses compared with imatinib and with lower rates of progression, especially in persons with Sokal intermediate- and high-risk scores [25-31]. However, this advantage for 2G-TKIs does not translate into better PFS or CML-related survival or survival. 2G-TKls are recommended for initial therapy of intermediate- and high-risk cohorts in the National Comprehensive Cancer Network (NCCN) clinical practice guidelines based on the risk of progression rather than PFS, CMLrelated survival, or survival [18]. This differs from the ELN 2020 recommendation that does not suggest a TKI preference based on risk cohort [17]. Complicating the NCCN recommendation is the question which predictive score should be used to classify someone as intermediate- or highrisk.

In our propensity-matching analyses, in subjects classified as intermediate risk using the Sokal or ELTS scores, we found that initial 2G-TKI therapy improved that proportions of CCyR, molecular responses, and FFS compared with initial imatinib therapy but not CML-related survival or survival. In subjects classified as intermediate risk using the ELTS but not the Sokal score, initial therapy with a 2G-TKI resulted in better PFS but not 
better CML-related survival or survival. This finding may influence TKI-therapy decisions for physicians focused on surrogate endpoints. Why 2G-TKls had no advantage in high-risk subjects identified by both scores could reflect relatively few subjects but also no favorable impact of $2 \mathrm{G}-\mathrm{TK}$ ls when disease biology is highly unfavorable.

Consistent with several studies, we found that females had better molecular responses to TKI therapy than males and lower probabilities of therapy failure and transformation to accelerated and blast phases [32-34]. This advantage might reflect different compliance or leukemia biology or other factors [35]. Similar to previous studies, we found a lower hemoglobin concentration and higher WBC counts were associated with worse responses and/or outcomes [36-39].

Our study has limitations. First, it is retrospective. Second, we lacked a validation cohort. Third, the number of subjects receiving initial 2G-TKI therapy was only 282. Fourth, 2G-TKIs were available only after 2011 resulting in an imbalance in follow-up. Also, therapy options for subjects failing imatinib before 2011 were restricted. Fifth, use of imatinib vs. a 2G-TKI was not random nor pre specified. As such, there are likely selection biases which we tried to account for propensity-score matching. We accept this is an imperfect simulation of a randomized controlled trial. Sixth, our subjects were younger than in most other CML studies in persons of predominantly European descent and need validation in these populations. Seventh, our data are from a specialized tertiary CML center with subjects coming from all over a large country. This obviously introduces subject-selection biases. Eighth, we did not consider other 2G-TKls approved for initial therapy, including bosutinib and radotinib. Whether our conclusions apply to these drugs is unknown. Ninth, we did not analyze interval to stopping TKI therapy or success rate of therapy-free remission. Last, we did not monitor adherence to TKI therapy which may have differed for different TKIs.

In conclusion, we found better overall prediction accuracy for the ELTS score compared with the Sokal score in persons with chronic-phase CML receiving TKI therapy, especially those receiving $2 \mathrm{G}$-TKls. People identified as intermediate risk in the ELTS score may benefit from 2G-TKI therapy compared with imatinib in achieving surrogate endpoints but not in CML-related survival or survival. The interval from start to stopping TKI-therapy and success rates of therapy-free remission were not compared.

\section{REFERENCES}

1. Hasford J, Pfirrmann M, Hehlmann R, Allan NC, Baccarani M, Kluin-Nelemans JC, et al. A new prognostic score for survival of patients with chronic myeloid leukemia treated with interferon alfa. writing committee for the collaborative CML prognostic factors project group. J Natl Cancer Inst. 1998;90:850-8.

2. Sokal JE, Cox EB, Baccarani M, Tura S, Gomez GA, Robertson JE, et al. Prognostic discrimination in "good-risk" chronic granulocytic leukemia. Blood. 1984;63:789-99.

3. Francis J, Dubashi B, Sundaram R, Pradhan SC, Chandrasekaran A. Influence of Sokal, Hasford, EUTOS scores and pharmacogenetic factors on the complete cytogenetic response at 1 year in chronic myeloid leukemia patients treated with imatinib. Med Oncol (Northwood, Lond, Engl). 2015;32:213.

4. Sinha SK, Sinha S, Mandal PK, Bhattacharyya NK, Pandey A, Gupta P. A comparative study of Hasford score and Sokal index in prognostication of the novo chronic myeloid leukemia patients and a search for new prognostic markers. Indian J Pathol Microbiol. 2013;56:216-20.

5. Uz B, Buyukasik $Y$, Atay $H$, Kelkitli E, Turgut $M$, Bektas $O$, et al. EUTOS CML prognostic scoring system predicts ELN-based 'event-free survival' better than Euro/Hasford and Sokal systems in CML patients receiving front-line imatinib mesylate. Hematol (Amst, Neth). 2013;18:247-52.

6. Xia L, Qian W, Yang M, Li Q, Liu F, Xie Y. Comparison of the utility and applicability of the Sokal, Hasford, and EUTOS scores in a population of Chinese patients with chronic-phase chronic myeloid leukemia undergoing imatinib therapy. OncoTargets Ther. 2015;8:2485-92.

7. Hasford J, Baccarani M, Hoffmann V, Guilhot J, Saussele S, Rosti G, et al. Predicting complete cytogenetic response and subsequent progression-free survival in 2060 patients with CML on imatinib treatment: the EUTOS score. Blood. 2011;118:686-92.

8. Pfirrmann M, Baccarani M, Saussele S, Guilhot J, Cervantes F, Ossenkoppele G, et al. Prognosis of long-term survival considering disease-specific death in patients with chronic myeloid leukemia. Leukemia. 2016;30:48-56.

9. Breccia M, Pregno P, Castagnetti F, Bonifacio M, Tiribelli M, Gozzini A, et al. EUTOS long-term survival score discriminates different Sokal score categories in chronic myeloid leukemia patients, showing better survival prediction. analysis of the GIMEMA CML observational study. Leukemia. 2021;35:1814-6.

10. Castagnetti F, Gugliotta G, Breccia M, Stagno F, Specchia G, Levato L, et al. The use of EUTOS long-term survival score instead of Sokal score is strongly advised in elderly chronic myeloid leukemia patients. Blood. 2018;132:44.

11. Geelen IGP, Sandin F, Thielen N, Janssen J, Hoogendoorn M, Visser O, et al. Validation of the EUTOS long-term survival score in a recent independent cohort of "real world" CML patients. Leukemia. 2018;32:2299-303.

12. Millot F, Guilhot J, Suttorp M, Güneş AM, Sedlacek P, De Bont E, et al. Prognostic discrimination based on the EUTOS long-term survival score within the International registry for chronic myeloid leukemia in children and adolescents. Haematologica. 2017;102:1704-8.

13. Molica M, Canichella M, Alunni Fegatelli D, Colafigli G, Massaro F, Latagliata R, et al. The EUTOS long-term survival score accurately predicts the risk of death in chronic myeloid leukaemia patients treated outside of clinical trials. Am J Hematol. 2017;92: E661-e4.

14. Pfirrmann M, Clark RE, Prejzner W, Lauseker M, Baccarani M, Saussele S, et al. The EUTOS long-term survival (ELTS) score is superior to the Sokal score for predicting survival in chronic myeloid leukemia. Leukemia. 2020;34:2138-49.

15. Sato E, Iriyama N, Tokuhira M, Takaku T, Ishikawa M, Nakazato T, et al. The EUTOS long-term survival score predicts disease-specific mortality and molecular responses among patients with chronic myeloid leukemia in a practice-based cohort. Cancer Med. 2020;9:8931-9.

16. Yang X, Bai Y, Shi M, Zhang W, Niu J, Wu C, et al. Validation of the EUTOS longterm survival score in Chinese chronic myeloid leukemia patients treated with imatinib: a multicenter real-world study. Cancer Manag Res. 2020;12:1293-301.

17. Hochhaus A, Baccarani M, Silver RT, Schiffer C, Apperley JF, Cervantes F, et al. European LeukemiaNet 2020 recommendations for treating chronic myeloid leukemia. Leukemia. 2020;34:966-84.

18. Deininger MW, Shah NP, Altman JK, Berman E, Bhatia R, Bhatnagar B, et al. Chronic myeloid leukemia, version 2.2021, NCCN clinical practice guidelines in oncology. J Natl Compr Cancer Netw: JNCCN. 2020;18:1385-415.

19. Baccarani M, Cortes J, Pane F, Niederwieser D, Saglio G, Apperley J, et al. Chronic myeloid leukemia: an update of concepts and management recommendations of European LeukemiaNet. J Clin Oncol: Off J Am Soc Clin Oncol. 2009;27: 6041-51.

20. Baccarani M, Deininger MW, Rosti G, Hochhaus A, Soverini S, Apperley JF, et al. European leukemiaNet recommendations for the management of chronic myeloid leukemia: 2013. Blood. 2013;122:872-84.

21. Baccarani M, Saglio G, Goldman J, Hochhaus A, Simonsson B, Appelbaum F, et al. Evolving concepts in the management of chronic myeloid leukemia: recommendations from an expert panel on behalf of the European leukemiaNet. Blood. 2006;108:1809-20.

22. Qin $Y Z$, Jiang $Q$, Jiang $H$, Li JL, Li LD, Zhu HH, et al. Which method better evaluates the molecular response in newly diagnosed chronic phase chronic myeloid leukemia patients with imatinib treatment, BCR-ABL(IS) or log reduction from the baseline level? Leuk Res. 2013;37:1035-40.

23. Lauseker M, Gerlach R, Worseg W, Haferlach T, Tauscher M, Hasford J, et al. Differences in treatment and monitoring of chronic myeloid leukemia with regard to age, but not sex: Results from a population-based study. Eur J Haematol. 2019;103:362-9.

24. Lokesh KN, Pehalajani JK, Loknatha D, Jacob LA, Babu MCS, Rudresha AH, et al. CML in elderly: does age matter? Indian J Hematol Blood Transfus. 2020;36:47-50.

25. Cortes JE, Gambacorti-Passerini C, Deininger MW, Mauro MJ, Chuah C, Kim DW, et al. Bosutinib Versus Imatinib for Newly Diagnosed Chronic Myeloid Leukemia: Results From the Randomized BFORE Trial. J Clin Oncol. 2018;36:231-7.

26. Cortes JE, Jiang Q, Wang J, Weng J, Zhu H, Liu X, et al. Dasatinib vs. imatinib in patients with chronic myeloid leukemia in chronic phase (CML-CP) who have not achieved an optimal response to 3 months of imatinib therapy: the DASCERN randomized study. Leukemia. 2020;34:2064-73.

27. Cortes JE, Saglio G, Kantarjian HM, Baccarani M, Mayer J, Boqué C, et al. Final 5-Year study results of DASISION: the dasatinib versus imatinib study in treatment-naïve chronic myeloid leukemia patients trial. J Clin Oncol. 2016; 34:2333-40.

28. Hochhaus A, Gambacorti-Passerini C, Abboud C, Gjertsen BT, Brümmendorf TH, Smith BD, et al. Bosutinib for pretreated patients with chronic phase chronic myeloid leukemia: primary results of the phase 4 BYOND study. Leukemia 2020; 34:2125-37. 
29. Hochhaus A, Saglio G, Hughes TP, Larson RA, Kim DW, Issaragrisil S, et al. Long-term benefits and risks of frontline nilotinib vs imatinib for chronic myeloid leukemia in chronic phase: 5-year update of the randomized ENESTnd trial. Leukemia. 2016;30:1044-54.

30. Kantarjian HM, Hughes TP, Larson RA, Kim DW, Issaragrisil S, le Coutre $P$, et al. Long-term outcomes with frontline nilotinib versus imatinib in newly diagnosed chronic myeloid leukemia in chronic phase: ENESTnd 10-year analysis. Leukemia. 2021:35:440-53.

31. Shah NP, Rousselot P, Schiffer C, Rea D, Cortes JE, Milone J, et al. Dasatinib in imatinib-resistant or -intolerant chronic-phase, chronic myeloid leukemia patients: 7-year follow-up of study CA180-034. Am J Hematol. 2016;91:869-74.

32. Branford S, Yeung DT, Ross DM, Prime JA, Field CR, Altamura HK, et al. Early molecular response and female sex strongly predict stable undetectable $B C R-A B L 1$, the criteria for imatinib discontinuation in patients with CML. Blood. 2013;121:3818-24.

33. Breccia M, Colafigli G, Molica M, De Angelis F, Quattrocchi L, Latagliata R, et al. Sex correlates with differences in long-term outcome in chronic myeloid leukaemia patients treated with imatinib. Br J Haematol. 2016;173:945-6.

34. Lin HX, Sjaarda J, Dyck J, Stringer R, Hillis C, Harvey M, et al. Gender and BCR-ABL transcript type are correlated with molecular response to imatinib treatment in patients with chronic myeloid leukemia. Eur J Haematol. 2016;96:360-6.

35. Marin D, Bazeos A, Mahon FX, Eliasson L, Milojkovic D, Bua M, et al. Adherence is the critical factor for achieving molecular responses in patients with chronic myeloid leukemia who achieve complete cytogenetic responses on imatinib. J Clin Oncol. 2010;28:2381-8.

36. Bewersdorf JP, Zeidan AM. Hyperleukocytosis and leukostasis in acute myeloid leukemia: can a better understanding of the underlying molecular pathophysiology lead to novel treatments? Cells. 2020;9:2310.

37. Dou X, Zheng F, Zhang L, Jin J, Zhang Y, Liu B, et al. Adolescents experienced more treatment failure than children with chronic myeloid leukemia receiving imatinib as frontline therapy: a retrospective multicenter study. Annals Hematol. 2021;100:2215-28.

38. Ko PS, Yu YB, Liu YC, Wu YT, Hung MH, Gau JP, et al. Moderate anemia at diagnosis is an independent prognostic marker of the EUTOS, Sokal, and Hasford scores for survival and treatment response in chronic-phase, chronic myeloid leukemia patients with frontline imatinib. Curr Med Res Opin. 2017;33:1737-44.

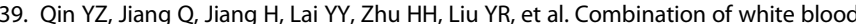
cell count at presentation with molecular response at 3 months better predicts deep molecular responses to imatinib in newly diagnosed chronic-phase chronic myeloid leukemia patients. Medicine 2016;95:e2486.

\section{ACKNOWLEDGEMENTS}

We thank medical staff and patient participants. RPG acknowledges support from the National Institute of Health Research (NIHR) Biomedical Research Centre funding scheme. This study was funded, in part, by the National Nature Science Foundation of China (No. 81770161, No. 81970140).

\section{AUTHOR CONTRIBUTIONS}

QJ and X-JH designed the study and provided subjects. QJ and X-SZ collected and analyzed the data. QJ, X-SZ, RPG, and X-JH prepared the typescript. All authors approved the final typescript, took responsibility, and agreed to submit for publication.

\section{COMPETING INTERESTS}

RPG is a consultant to BeiGene Ltd., Fusion Pharma LLC, LaJolla NanoMedical Inc. Mingsight Parmaceuticals Inc., and CStone Pharmaceuticals; advisor to Antegene Biotech LLC, medical director, FFF Enterprises Inc.; partner, AZAC Inc.; Board of Directors, Russian Foundation for Cancer Research Support; and Scientific Advisory Board: StemRad Ltd.

\section{ETHICS APPROVAL}

The study was approved by the Ethics Committee of People's Hospital Beijing compliant with the Helsinki Declaration.

\section{ADDITIONAL INFORMATION}

Supplementary information The online version contains supplementary material available at https://doi.org/10.1038/s41375-021-01387-y.

Correspondence and requests for materials should be addressed to X.-J.H. or Q.J.

Reprints and permission information is available at http://www.nature.com/ reprints

Publisher's note Springer Nature remains neutral with regard to jurisdictional claims in published maps and institutional affiliations.

\begin{abstract}
Open Access This article is licensed under a Creative Commons Attribution 4.0 International License, which permits use, sharing, daptation, distribution and reproduction in any medium or format, as long as you give appropriate credit to the original author(s) and the source, provide a link to the Creative Commons license, and indicate if changes were made. The images or other third party material in this article are included in the article's Creative Commons license, unless indicated otherwise in a credit line to the material. If material is not included in the article's Creative Commons license and your intended use is not permitted by statutory regulation or exceeds the permitted use, you will need to obtain permission directly from the copyright holder. To view a copy of this license, visit http://creativecommons. org/licenses/by/4.0/.
\end{abstract}

(c) The Author(s) 2021 